\title{
Maximization of Gain in Slow-Light Silicon Raman Amplifiers
}

\author{
Ivan D. Rukhlenko, ${ }^{1}$ Malin Premaratne, ${ }^{1}$ and Govind P. Agrawal ${ }^{2}$ \\ ${ }^{1}$ Advanced Computing and Simulation Laboratory, Monash University, Clayton, VIC 3800, Australia \\ ${ }^{2}$ The Institute of Optics, University of Rochester, Rochester, NY 14627, USA
}

Correspondence should be addressed to Ivan D. Rukhlenko, rukhlenko.ivan@gmail.com

Received 12 February 2011; Accepted 26 April 2011

Academic Editor: Kazumi Wada

Copyright (C) 2011 Ivan D. Rukhlenko et al. This is an open access article distributed under the Creative Commons Attribution License, which permits unrestricted use, distribution, and reproduction in any medium, provided the original work is properly cited.

\begin{abstract}
We theoretically study the problem of Raman gain maximization in uniform silicon photonic-crystal waveguides supporting slow optical modes. For the first time, an exact solution to this problem is obtained within the framework of the undepleted-pump approximation. Specifically, we derive analytical expressions for the maximum signal gain, optimal input pump power, and optimal length of a silicon Raman amplifier and demonstrate that the ultimate gain is achieved when the pump beam propagates at its maximum speed. If the signal's group velocity can be reduced by a factor of 10 compared to its value in a bulk silicon, it may result in ultrahigh gains exceeding $100 \mathrm{~dB}$. We also optimize the device parameters of a silicon Raman amplifier in the regime of strong pump depletion and come up with general design guidelines that can be used in practice.
\end{abstract}

\section{Introduction}

The rapid progress in the field of silicon photonics realized during recent years draws the reality of all-optical signal processing on a nanoscale closer than ever [1-3]. Of paramount importance for the integration of optical functionalities on a silicon platform is the problem of efficient signal amplification through stimulated Raman scattering (SRS) [4-9]. Even though the Raman gain coefficient is relatively large for the silicon material, its use for signal amplification is hampered considerably by its large two-photon absorption (TPA) coefficient $\left(\sim 0.5 \mathrm{GW} / \mathrm{cm}^{2}\right)$ in the wavelength region near $1550 \mathrm{~nm}$ [10] and the effects of free-carrier absorption (FCA) that come into play once free carriers are generated via TPA.

Several techniques have been successfully employed to mitigate the nonlinear losses and increase the Raman gain in silicon-on-insulator (SOI) waveguides. The most straightforward method lies in the reduction of free-carrier lifetime. It is typically implemented through a reverse-bias $\mathrm{p}-\mathrm{i}-\mathrm{n}$ diode, which uses silicon waveguide as an intrinsic region and removes carriers from the region near its mode center through the applied static electric field [11-13]. This approach has been used to make silicon Raman amplifiers (SRAs) operating continuously. In the pulsed regime, in ad- dition to gain enhancement, this technique allows operation of an SRA at a bit rate as high as $100 \mathrm{Gbit} / \mathrm{s}$. Once free carriers are efficiently removed and they stop affecting optical propagation, TPA becomes the only nonlinear effect that limits performance of an SRA [14].

Signal gain can also be improved by successively optimizing both the length of the SOI waveguide and the input pump intensity $[15,16]$. Existence of the unique length that provides the maximum gain for given material parameters is evident from the following consideration. If the waveguide is too short, signal does not have enough time to build up its energy through interaction with the pump. In a very long waveguide, on the other hand, the gain approaches zero, as the signal is constantly exposed to TPA and linear losses that decay with optical intensity slower than the SRS does. This means that the silicon waveguide should end at the point where the gain peaks. Inside an SRA of optimal length, the signal gain can still have a local minimum, in the event that FCA-induced energy dissipation predominates signal amplification near the input facet of the waveguide. If that is the case, one can increase the gain by reducing the intensity of the input pump beam. The existence of an optimal pump intensity for a fixed length of SRA can also be understood with a similar argument. 
Another way of enhancing the amplified signal lies in engineering (tapering) the lateral cross-section of a SRA [17-22]. The objective of tapering is to provide an optimal balance between the nonlinear losses and SRS, which is possible because they scale differently with the effective mode area. By developing a semianalytical method for calculating the optimal tapering profile of an SRA, we have shown that linear tapering of the amplifier is capable of increasing gain almost up to its ultimate limit that is reached with an irregular optimal tapering [17].

A somewhat more challenging, but quite efficient, gainimprovement technique relies on the slow-light enhancement of nonlinear optical phenomena in silicon photonic crystal (SPhC) waveguides [23-29]. A reduction in the group velocities of the pump and signal beams inside a $\mathrm{SPhC}$ waveguide leads to an increase in beams intensities and allows them to stay longer inside the amplifier, thereby improving the efficiency of the SRS process. This speed reduction, unfortunately, also results in the enhancement of TPA and FCA, making the problem of gain maximization in slow-light SRAs more intricate compared to that in ordinary SRAs. The only attempt to analyze gain scaling with group velocity and find the optimal parameters for a SPhCbased SRA belongs to Krause et al. [30]. In this paper, we extend this study and present an exact solution to the problem of gain maximization in slow-light SRAs within the undepleted-pump approximation. We also perform a numerical optimization of various SRA parameters in the regime of strong pump depletion, in order to come up with the design guidelines that can be applied in practice.

\section{Formulation of the Problem}

Consider amplification of a continuous wave (CW) signal at the frequency $\omega_{s}$ by a CW pump at the frequency $\omega_{p}$ inside a $\mathrm{SPhC}$ waveguide, which is characterized by the slow-down factors $S_{s}$ and $S_{p}$ at frequencies $\omega_{s}$ and $\omega_{p}$, respectively. If the pump copropagates with the signal, then their "sloweddown" intensities $Q_{p(s)}(z)=S_{p(s)} I_{p(s)}(z)$, where $I_{p(s)}(z)$ are the ordinary intensities, satisfy the following system of coupled differential equations $[23,30]$ :

$$
\begin{aligned}
\frac{1}{S_{p} Q_{p}} \frac{\mathrm{d} Q_{p}}{\mathrm{~d} z}= & -\alpha_{p}-\beta_{p} Q_{p}-\gamma_{p} Q_{s} \\
& -\xi_{p}\left[Q_{p}^{2}+4 Q_{p} Q_{s}+\left(\omega_{p} / \omega_{s}\right) Q_{s}^{2}\right] \\
\frac{1}{S_{s} Q_{s}} \frac{\mathrm{d} Q_{s}}{\mathrm{~d} z}= & -\alpha_{s}-\beta_{s} Q_{s}+\gamma_{s} Q_{p} \\
& -\xi_{s}\left[Q_{p}^{2}+4 Q_{p} Q_{s}+\left(\omega_{p} / \omega_{s}\right) Q_{s}^{2}\right] .
\end{aligned}
$$

In these equations, the terms characterized by the coefficients $\alpha_{p(s)}, \beta_{p(s)}$, and $\xi_{p(s)}$ account for linear losses, TPA, and FCA of the pump and signal beams, respectively. The FCA coefficients are defined as

$$
\xi_{p(s)}=\frac{\sigma_{0}\left(\omega_{0} / \omega_{p(s)}\right)^{2} \tau_{c} \beta_{p}}{2 \hbar \omega_{p}}
$$

where $\sigma_{0}=1.45 \times 10^{-21} \mathrm{~m}^{2}, \omega_{0}=2 \pi c /(1.55 \mu \mathrm{m}), \tau_{c}$ is the effective free-carrier lifetime, and $\hbar$ is the reduced Planck's constant. The nonlinear parameters, $\gamma_{p}$ and $\gamma_{s}$, have been defined such that they describe both the cross-TPA and SRS effects. Assuming that the pump frequency exceeds the signal frequency exactly by the Raman shift $\omega_{p}-\omega_{s}=\Omega_{\mathrm{R}} \approx$ $(2 \pi) 15.6 \mathrm{THz}$, we have

$$
\gamma_{p}=g_{\mathrm{R}}+2 \beta_{p}, \quad \gamma_{s}=\frac{\left(g_{\mathrm{R}}-2 \beta_{s}\right)}{\eta},
$$

where $g_{\mathrm{R}}$ is the Raman gain coefficient and $\eta=\omega_{p} / \omega_{s}$.

Equations (1) are similar in form to the coupled-intensity equations obtained without the slow-light effects [31-33], but they contain two additional parameters $S_{p}$ and $S_{s}$. Strictly speaking, these parameters are related to each other, since they both depend on geometric and material characteristics of the same photonic crystal. However, owing to a great number of such characteristics and the possibility of varying most of them in different ways within wide limits, we may think of the slow-down factors for pump and signal modes as independent of each other. These factors can be adjusted to take different value (from 1 to as large as 1000) by engineering the dispersion of the SPhC waveguide $[29,34]$. We also assume that the input pump intensity $I_{p 0}$ may take any value between zero and infinity because it can be varied by changing either the pump power or the SRA cross-section.

Hence, the problem of gain maximization in a slow-light SRA, for a given intensity of the input signal, consists of searching for the optimal values of the waveguide length $L$, input pump intensity $I_{p 0}$, and slow-down factors $S_{s}$ and $S_{p}$. If some of these parameters are fixed by practical needs or restricted by fabrication capability, the optimization problem becomes simpler because it involves fewer than four parameters.

\section{Exact Solution: Undepleted-Pump Approximation}

The signal gain can be readily optimized analytically in the undepleted-pump regime, which usually holds for relatively short SRAs with $g_{\mathrm{R}} \lesssim 10 \mathrm{GW} / \mathrm{cm}^{2}$ and for low input signal powers. We now consider this scenario in detail, for it is quite instructive and helps us to illuminate the peculiarities of the optimization problem in the general case.

In the approximation of an undepleted pump, valid as long as $Q_{p} \gg Q_{s}$ everywhere inside the $\mathrm{SPhC}$ waveguide, (1) reduces to

$$
\begin{gathered}
\frac{1}{S_{p} Q_{p}} \frac{\mathrm{d} Q_{p}}{\mathrm{~d} z} \approx-\alpha_{p}-\beta_{p} Q_{p}-\xi_{p} Q_{p}^{2}, \\
\frac{1}{S_{s} Q_{s}} \frac{\mathrm{d} Q_{s}}{\mathrm{~d} z} \approx-\alpha_{s}+\gamma_{s} Q_{p}-\xi_{s} Q_{p}^{2} .
\end{gathered}
$$

Equation (4b) shows that the signal gain grows with increasing $S_{s}$, confirming that one can improve the performance of an SRA by slowing down the signal mode as much as possible [30]. The situation is less clear with the optimal 
slow-down factor for the pump. Indeed, when the input pump intensity is fixed, an increase in the time the pump spends inside the amplifier may result in either increase or decrease in the value of $Q_{p}$, which, in turn, may enhance or reduce the local signal gain. Therefore, the three parameters to be optimized in the undepleted-pump regime are the amplifier length, the input pump intensity, and the slow-down factor for the pump field.

To find the values of the three optimal parameters, we use the implicit solution of (4a) and (4b). It can be written compactly with the shortened notations $Q_{0} \equiv Q_{p}(0)$ and $Q_{L} \equiv Q_{p}(L)$ in the form of the following coupled equations [35]:

$$
\begin{aligned}
\frac{\alpha_{p}+\beta_{p} Q_{L}+\xi_{p} Q_{L}^{2}}{\alpha_{p}+\beta_{p} Q_{0}+\xi_{p} Q_{0}^{2}} & =\frac{Q_{L}^{2}}{Q_{0}^{2}} \exp \left(2 \alpha_{p} S_{p} L+\beta_{p} Q_{0} L_{\text {eff }}\right), \\
L_{\text {eff }} & =\frac{\mu}{\beta_{p} Q_{0}} \ln \left(\frac{\mu F_{L}+1}{\mu F_{L}-1} \frac{\mu F_{0}-1}{\mu F_{0}+1}\right),
\end{aligned}
$$

where $\mu=\left(1-4 \alpha_{p} \xi_{p} / \beta_{p}^{2}\right)^{-1 / 2}, F_{j}=1+2 \xi_{p} Q_{j} / \beta_{p}$, and $L_{\text {eff }}$ is the effective length of the amplifier.

Using (4b), (5a), and (5b), it is easy to show that the signal gain $G_{s}$ is given by the relation

$$
\begin{aligned}
\left(\frac{S_{p}}{S_{s}}\right) \ln G_{s}= & -\left(\alpha_{s}-\eta^{2} \alpha_{p}\right) S_{p} L \\
& +\left(\gamma_{s}+\eta^{2} \beta_{p}\right) Q_{0} L_{\text {eff }}-\eta^{2} \ln \left(\frac{Q_{0}}{Q_{L}}\right) .
\end{aligned}
$$

We maximize $G_{s}$ with respect to the three optimization parameters, $L, I_{p 0}$, and $S_{p}$, by requiring that the following three derivatives vanish:

$$
\begin{aligned}
& \frac{\partial G_{s}\left(L, I_{p 0}, S_{p}\right)}{\partial L}=0, \\
& \frac{\partial G_{s}\left(L, I_{p 0}, S_{p}\right)}{\partial I_{p 0}}=0, \\
& \frac{\partial G_{s}\left(L, I_{p 0}, S_{p}\right)}{\partial S_{p}}=0 .
\end{aligned}
$$

Upon employing (4b)-(6), these requirements lead to the following three relations:

$$
\begin{aligned}
\alpha_{s}-\gamma_{s} Q_{L}+\xi_{s} Q_{L}^{2} & =0, \\
Q_{0}+Q_{L}= & \frac{\gamma_{s}}{\xi_{s}}, \\
\frac{S_{p}}{S_{s}} \ln G_{s}= & \frac{\gamma_{s}-\xi_{s}\left(Q_{0}+Q_{L}\right)}{\alpha_{p}+\beta_{p} Q_{0}+\xi_{p} Q_{0}^{2}}\left(Q_{0}-Q_{L}\right) \\
& -\left(\alpha_{s}-\gamma_{s} Q_{L}+\xi_{s} Q_{L}^{2}\right) S_{p} L .
\end{aligned}
$$

The combination of $(8 \mathrm{a})-(8 \mathrm{c})$ leads to the equality $\ln G_{s}=0$, which is in conflict with (6). This implies that not more than two of the parameters $L, I_{p 0}$, and $S_{p}$ can be optimized at the same time. Next, substitution of (8b) into (8c) results in

$$
\ln G_{s}=-\left(\alpha_{s}-\gamma_{s} Q_{L}+\xi_{s} Q_{L}^{2}\right) S_{s} L .
$$

This equation is not satisfied in real SRAs since, according to (4b), it requires pump power to be constant along the amplifier. Consequently, the gain provided by an SRA of a given length peaks simultaneously with either the slowdown factor $S_{p}$ or the input pump intensity. The optimal parameters of an SRA depend on the optimization scenario. In what follows, we consider the three possible optimization schemes.

3.1. Optimal $L$ and $I_{p 0}$ for a Fixed $S_{p}$. When the slow-down factor of the pump is fixed, (8a) and (8b) yield the critical values for the quantities $Q_{0}$ and $Q_{L}$ of the form

$$
Q_{0}=\frac{\gamma_{s}+\sqrt{\gamma_{s}^{2}-4 \alpha_{s} \xi_{s}}}{2 \xi_{s}}, \quad Q_{L}=\frac{\gamma_{s}-\sqrt{\gamma_{s}^{2}-4 \alpha_{s} \xi_{s}}}{2 \xi_{s}} .
$$

It is evident from these expressions that the inequality $\gamma_{s}^{2}>$ $4 \alpha_{s} \xi_{s}$ must be satisfied to allow for amplification of signal. This inequality sets the upper limit for the product $\alpha_{s} \tau_{c}$ [30]. For $\gamma_{s}^{2}>4 \alpha_{s} \xi_{s}$, one can see from ( $\left.4 \mathrm{~b}\right)$ that the derivative $\mathrm{d} Q_{s} / \mathrm{d} z$ remains positive as long as $Q_{0}>Q_{p}>Q_{L}$. The expressions in (10) simply determine the pump intensities corresponding to the onset and the end of local net gain and thus enable signal amplification along the whole length of a $\mathrm{SPhC}$ waveguide.

With the initial and final intensities given by (10), the optimal length of the SRA can be explicitly found from (5a) while the optimal input intensity of the pump is simply given by $I_{p 0}=Q_{0} / S_{p}$.

It is important to note that the optimal values of $Q_{0}$ and $Q_{L}$ do not depend on the parameter $S_{p}$. Assuming that the signal exhibits a net gain, we conclude from (6) that the maximum signal gain corresponds to the lowest possible value of $S_{p}$, that is, to $S_{p}=1$. According to $(4 \mathrm{a})$, this value results in the slowest attenuation of the pump along the amplifier.

Hence, the optimal values of three parameters that maximize the signal gain for this optimization scheme are given by

$$
\begin{gathered}
I_{p 0}=Q_{0}, \quad S_{p}=1, \\
L=\frac{1}{2 \alpha_{p}}\left[\ln \left(\frac{Q_{0}^{2}}{Q_{L}^{2}} \frac{\alpha_{p}+\beta_{p} Q_{L}+\xi_{p} Q_{L}^{2}}{\alpha_{p}+\beta_{p} Q_{0}+\xi_{p} Q_{0}^{2}}\right)-\beta_{p} Q_{0} L_{\mathrm{eff}}\right],
\end{gathered}
$$

where $Q_{0}$ and $Q_{L}$ are given by (10).

3.2. Optimal $L$ and $S_{p}$ for a Fixed $I_{p 0}$. If we keep the input pump power constant, then (8a) yields the optimal value for $Q_{L}$ given in (10), where the minus before the radical is chosen to provide the maximal signal gain. With this value, the critical value of $Q_{0}>Q_{L}$ is found by simultaneously solving (5a), (5b), (6), and (8c). It should be noted that $Q_{0}$ 


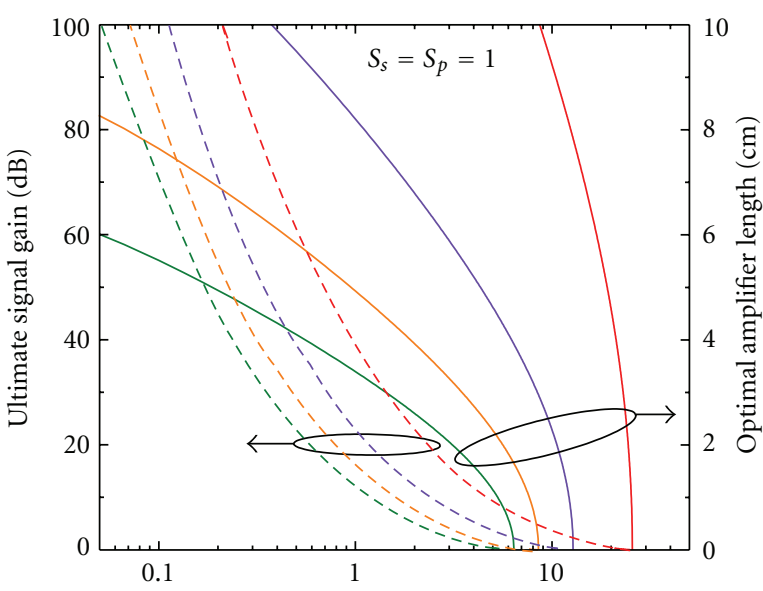

(a)
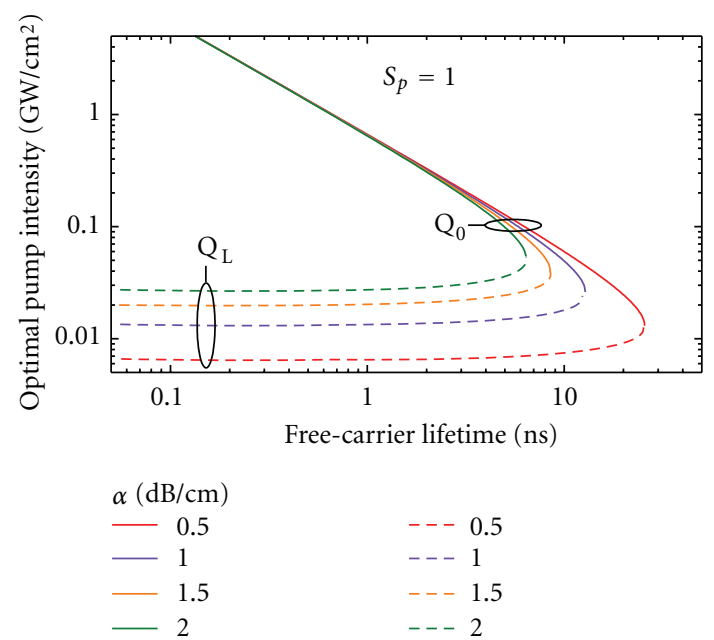

(b)

Figure 1: (a) Ultimate signal gain (dashed curves) and optimal amplifier length (solid curves) plotted as functions of $\tau_{c}$ for an SRA operating in the undepleted-pump regime when $S_{s}=S_{p}=1$; different colors correspond to different values of $\alpha_{s}=\alpha_{p} \equiv \alpha$. (b) Input and output pump intensities calculated as a function of $\tau_{c}$ using (10) with $S_{p}=1$. Refer to text for other parameters.

obtained in this way does not generally coincide with that in (10).

Once the optimal quantities $Q_{0}$ and $Q_{L}$ are known, the optimal slow-down factor $S_{p}$ is given by $S_{p}=Q_{0} / I_{p 0}$ and the optimal length of the SRA is calculated from (5a).

On substitution of (8a) into (8c), the signal gain may be written as

$$
\ln G_{s}=\frac{\gamma_{s}-\xi_{s}\left(Q_{0}+Q_{L}\right)}{\alpha_{p}+\beta_{p} Q_{0}+\xi_{p} Q_{0}^{2}}\left(\frac{Q_{0}}{Q_{L}}-1\right) S_{s} I_{p 0} .
$$

This expression shows that the peak net gain grows in proportion to the input pump power. Since the maximum pump power is equal to $Q_{0}$ when $S_{p}=1$, we again arrive at the set of optimal parameters given in (11a) and (11b).

3.3. Optimal $I_{p 0}$ and $S_{p}$ for a Fixed L. Consider finally the situation in which the length of an SRA is preset. As we have seen above, in this case (8b) and (8c) are inconsistent. Therefore, one may either find the optimal input pump power, $I_{p 0}\left(S_{p}, L\right)$, for given values of $S_{p}$ and $L$ by solving $(5 a),(5 b)$, and $(8 b)$ or the optimal slow-down factor of the pump, $S_{p}\left(I_{p 0}, L\right)$, for given values of $I_{p 0}$ and $L$ by solving ( $\left.5 \mathrm{a}\right)$, (5b), and (8c). Of course, if after that we also optimize the length of the amplifier, we arrive at the results obtained for the previous two optimization schemes.

Since the values of $Q_{0}$ obtained with different optimization schemes are different, the optimal amplifier length and input pump power in (11a) and (11b) are also different. From physical considerations it is, however, clear that the optimal values of $Q_{0}$ and $Q_{L}$ providing the ultimate signal gain are those given in (10).

In Figure 1, we plot the maximum signal gain as a function of free-carrier lifetime that can be achieved in the undepleted-pump regime with a "fast" signal mode $\left(S_{s}=\right.$ 1). The same figure shows the optimal SRA length and the optimal pump intensity required for the maximum gain. The material parameters are chosen to be $\lambda_{s}=1.55 \mu \mathrm{m}, \lambda_{p}=$ $1.434 \mu \mathrm{m}, \alpha_{s}=\alpha_{p}=0.5-2 \mathrm{~dB} / \mathrm{cm}, \beta_{s}=\beta_{p}=0.5 \mathrm{GW} / \mathrm{cm}^{2}$, and $g_{\mathrm{R}}=20 \mathrm{GW} / \mathrm{cm}^{2}$. It is seen that the signal gains of $>20 \mathrm{~dB}$ can be realized with pump powers below $1 \mathrm{GW} / \mathrm{cm}^{2}$ if $\tau_{c} \lesssim 1$ ns and linear losses do not exceed $1 \mathrm{~dB} / \mathrm{cm}$. These gains can be increased further by a factor of $S_{s}$, which can be more than an order of magnitude, provided the signal beam is sufficiently slowed down by dispersion engineering. Unfortunately, such strong amplification requires relatively long $\mathrm{SPhC}$ waveguides (longer than $8 \mathrm{~cm}$ in the above example).

Equations (6) and (11b) show that the length of a SRA can be reduced to a few micrometers without noticeably affecting the gain, by simultaneously increasing linear losses and reducing free-carrier absorption in such a manner that the product $\alpha_{s} \tau_{c}$ remains constant (so that the values of $Q_{0}$ and $Q_{L}$ do not change). For instance, gains in excess of $20 \mathrm{~dB}$ (for $S_{s}=1$ ) can be achieved in a $1.5 \mathrm{~mm}$ long SRA with $\tau_{c}=0.02 \mathrm{~ns}$ and $\alpha_{s}=50 \mathrm{~dB} / \mathrm{cm}$. The optimum length of a SRA also becomes shorter when the amplifier operates in the regime of considerable pump depletion, which we analyze in the next section.

\section{Numerical Solution: General Case}

As we saw in the previous section, in the undepleted-pump regime, the signal mode should be slowed down as much as possible, while the pump mode should travel with the highest speed. This is not the case when the intensity of input signal, $I_{s 0}$, approaches the ratio $Q_{L} /\left(S_{s} G_{s}\right)$ and the pump's depletion can no longer be ignored [36]. The examples below illustrate the peculiarities of SRA optimization in the regime of heavy pump depletion, in which signal gain is calculated numerically from (1).

Figure 2 shows the contour plots of the net signal gain in an $8 \mathrm{~mm}$ long SRA for $I_{p 0}=30 \mathrm{MW} / \mathrm{cm}^{2}$ and $I_{s 0}=$ $0.1 \mathrm{MW} / \mathrm{cm}^{2}$; the depletion [37] of the pump beam at the amplifier output equals $76 \%$. It is seen that the maximum gain of approximately $17.7 \mathrm{~dB}$ is achieved for the slow-down factors $S_{s} \approx 7.8$ and $S_{p} \approx 3.4$. More importantly, this gain 


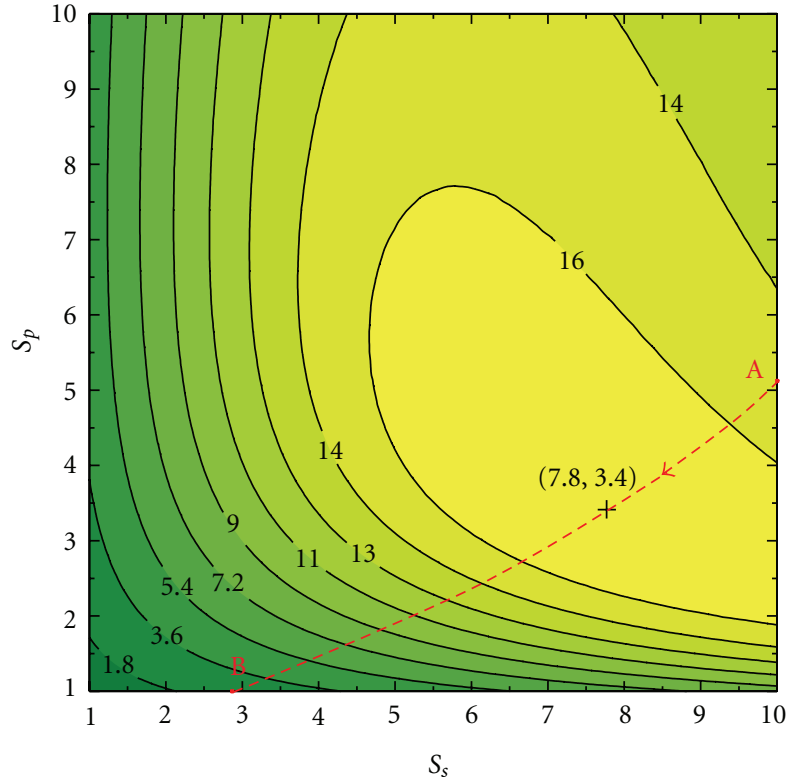

Figure 2: Contour plots of signal gain (in $\mathrm{dB}$ ) for an $8 \mathrm{~mm}$ long SRA in the plane formed by the factors $S_{s}$ and $S_{p}$ varying in the range 1 to 10 . The location marked by + corresponds to the peak gain of $17.7 \mathrm{~dB}$. Here, $I_{p 0}=30 \mathrm{MW} / \mathrm{cm}^{2}, I_{s 0}=0.1 \mathrm{MW} / \mathrm{cm}^{2}, \alpha=1 \mathrm{~dB} / \mathrm{cm}$, and $\tau_{c}=0.2 \mathrm{~ns}$. Other parameters are the same as in Figure 1 . The red curve shows how the gain peak's position drifts as $I_{p 0}$ changes from $15 \mathrm{MW} / \mathrm{cm}^{2}$ at point $\mathrm{A}(10,5.13)$ to $300 \mathrm{MW} / \mathrm{cm}^{2}$ at point $\mathrm{B}$ $(2.9,1)$.

value exceeds the maximum gain of $0.85 \mathrm{~dB}$ that occurs in the absence of the slow-light effects $\left(S_{s}=S_{p}=1\right)$ by more than a factor of 20. It should also be noted that, owing to a relatively flat gain peak, the range of tolerable slow-down parameters is rather broad, a feature that facilitates the design of SRAs.

The gain corresponding to the optimal slow-down factors can be increased by optimizing the input pump power. Red curves in Figures 2 and 3 show how the peak gain and the optimal factors $S_{s}$ and $S_{p}$ vary with $I_{p 0}$ for the same SRA of $8 \mathrm{~mm}$ length. One can see that the maximum peak gain of $31.7 \mathrm{~dB}$ is reached for $I_{p 0} \approx 3.5 \mathrm{GW} / \mathrm{cm}^{2}, S_{s} \approx 1.6$, and $S_{p}=$ 1. Although the obtained values of $I_{p 0}$ and $S_{p}$ coincide with those in the undepleted-pump regime, the maximum gain and the optimal amplifier length differ from the predictions in Figure $1(114 \mathrm{~dB}$ and $11 \mathrm{~cm}$, resp.). This is a consequence of strong pump depletion (about $84 \%$ in the present case).

A further enhancement of gain is generally possible by adjusting the length of the SPhC waveguide. Figure 3 shows this possibility by varying the amplifier length in the range of 2 to $12 \mathrm{~mm}$. However, as the gain profiles corresponding to 2,4 , and $12 \mathrm{~mm}$ long SRAs suggest, $L \approx 8 \mathrm{~mm}$ is the optimal length of the amplifier for this specific set of material parameters. For a typical effective mode area of $0.35 \mu \mathrm{m}^{2}$, pump power of $10 \mathrm{~W}$ is required at the input of the optimized SRA. Since it is very difficult to get such high input power levels in a CW regime, the actual maximum gain will be lower than $31.7 \mathrm{~dB}$ and will be set by the available pump power.

Comparison of different curves in Figure 3 leads to several conclusions that are important for designing SRAs with optimum performance. First, the optimum slow-down

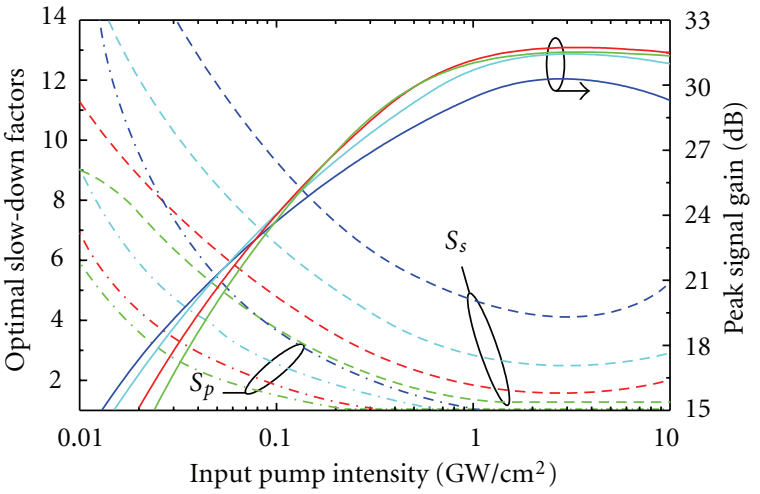

$L(\mathrm{~mm})$

2
$-\quad 8$

FIGURE 3: Optimal peak signal gain (solid curves) and slow-down factors, $S_{s}$ (dashed curves) and $S_{p}$ (dash-dotted curves), as functions of input pump intensity in a 2, 4, 8 , and $12 \mathrm{~mm}$ long SRAs. Other parameters are the same as in Figure 2.

factors for pump and signal can be reduced by increasing amplifier's length, because SRS is more efficient in longer waveguides. Second, as long as the ultimate signal gain is not achieved, larger pump intensities can compensate for operating with faster pump and signal modes. This is possible, since the reduction in time that light spends inside the amplifier is canceled by its stronger interaction with silicon. Third, strong FCA occurring at high intensities reduces pump depletion and, as a result, requires the use of a slowed signal mode (see the blue dashed curve). This regime is to be avoided in practice because the same peak gain can be achieved with a smaller pump intensity.

As a concluding remark, it is worth noting that our results are valid not only for SPhC waveguides, but also for any other types of slow-light silicon waveguides, regardless of the relative group delay that occurs upon light propagation through them.

\section{Conclusions}

We have theoretically analyzed the problem of gain maximization in silicon Raman amplifiers operating with slowlight pump and signal modes. Using the undepleted-pump approximation, we derived expressions for the maximum signal gain, optimal input pump power, and optimal length of the amplifier with a fixed cross-section. We also showed that the signal gain is maximized when the pump beam moves at its maximum speed. The signal gain grows indefinitely and can exceed $100 \mathrm{~dB}$, provided the signal beam travels more than 10 times slower than its speed in a bulk silicon. In the regime of substantial pump depletion, the parameters of an amplifier were optimized numerically and a relatively large CW gain of $31.7 \mathrm{~dB}$ was predicted for an $8 \mathrm{~mm}$ long waveguide pumped at an intensity of $3.5 \mathrm{GW} / \mathrm{cm}^{2}$. This result suggests that optimization of slow-light silicon photonic crystal waveguides is a promising approach to the 
realization of highly efficient Raman amplifiers built using the SOI platform.

\section{Acknowledgments}

This work was supported by the Australian Research Council, through its Discovery Grant scheme under grants nos. DP0877232 and DP110100713. The work of G. P. Agrawal is also supported by the US National Science Foundation award ECCS-0801772.

\section{References}

[1] J. Leuthold, C. Koos, and W. Freude, "Nonlinear silicon photonics," Nature Photonics, vol. 4, no. 8, pp. 535-544, 2010.

[2] R. Soref, "The past, present, and future of silicon photonics," IEEE Journal on Selected Topics in Quantum Electronics, vol. 12, no. 6, pp. 1678-1687, 2006.

[3] G. T. Reed and A. P. Knights, Silicon Photonics: An Introduction, John Wiley \& Sons, Hoboken, NJ, USA, 2004.

[4] M. A. Foster, A. C. Turner, J. E. Sharping, B. S. Schmidt, M. Lipson, and A. L. Gaeta, "Broad-band optical parametric gain on a silicon photonic chip," Nature, vol. 441, no. 7096, pp. 960-963, 2006.

[5] B. Jalali, V. Raghunathan, D. Dimitropoulos, and O. Boyraz, "Raman-based silicon photonics," IEEE Journal on Selected Topics in Quantum Electronics, vol. 12, no. 3, pp. 412-421, 2006.

[6] R. Jones, H. Rong, A. Liu et al., "Net continuous wave optical gain in a low loss silicon-on-insulator waveguide by stimulated Raman scattering," Optics Express, vol. 13, no. 2, pp. 519-525, 2005.

[7] O. Boyraz and B. Jalali, "Demonstration of $11 \mathrm{~dB}$ fiber-to-fiber gain in a silicon Raman amplifier," IEICE Electronics Express, vol. 1, pp. 429-434, 2004.

[8] A. Liu, H. Rong, M. Paniccia, O. Cohen, and D. Hak, "Net optical gain in a low loss silicon-on-insulator waveguide by stimulated Raman scattering," Optics Express, vol. 12, no. 18, pp. 4261-4268, 2004.

[9] R. Claps, D. Dimitropoulos, V. Raghunathan, Y. Han, and B. Jalali, "Observation of stimulated Raman amplification in silicon waveguides," Optics Express, vol. 11, no. 15, pp. 17311739, 2003.

[10] H. K. Tsang and Y. Liu, "Nonlinear optical properties of silicon waveguides," Semiconductor Science and Technology, vol. 23, no. 6, Article ID 064007, 2008.

[11] T. K. Liang and H. K. Tsang, "Nonlinear absorption and Raman scattering in silicon-on-insulator optical waveguides," IEEE Journal on Selected Topics in Quantum Electronics, vol. 10, no. 5, pp. 1149-1153, 2004.

[12] R. Claps, V. Raghunathan, D. Dimitropoulos, and B. Jalali, "Influence of nonlinear absorption on Raman amplification in Silicon waveguides," Optics Express, vol. 12, no. 12, pp. 27742780, 2004.

[13] T. K. Liang and H. K. Tsang, "Role of free carriers from two-photon absorption in Raman amplification in silicon-oninsulator waveguides," Applied Physics Letters, vol. 84, no. 15, pp. 2745-2747, 2004.

[14] H. Renner, "Silicon Raman amplifiers in the limit of zero free-carrier lifetime," in Proceedings of the IEEE International Conference on Group IV Photonics (GFP '07), pp. 137-139, 2007, paper WP30.
[15] M. Krause, H. Renner, and E. Brinkmeyer, "Silicon Raman amplifiers with ring-resonator-enhanced pump power," IEEE Journal on Selected Topics in Quantum Electronics, vol. 16, no. 1, pp. 216-225, 2010.

[16] H. Renner and M. Krause, "Maximal total gain of nontapered silicon-on-insulator Raman amplifiers," in Proceedings of the Optical Amplifiers and Their Applications Topical Meeting (OAA '06), 2006, paper OMD2.

[17] I. D. Rukhlenko, C. Dissanayake, M. Premaratne, and G. P. Agrawal, "Optimization of Raman amplification in silicon waveguides with finite facet reflectivities," IEEE Journal on Selected Topics in Quantum Electronics, vol. 16, no. 1, pp. 226233, 2010.

[18] I. D. Rukhlenko, C. Dissanayake, M. Premaratne, and G. P. Agrawal, "Maximization of net optical gain in siliconwaveguide Raman amplifiers," Optics Express, vol. 17, no. 7, pp. 5807-5814, 2009.

[19] M. Krause, H. Renner, and E. Brinkmeyer, "Efficient Raman lasing in tapered silicon waveguides," Spectroscopy, vol. 21, no. 1, pp. 26-32, 2006.

[20] H. Renner, M. Krause, and E. Brinkmeyer, "Maximal gain and optimal taper design for Raman amplifiers in siliconon-insulator waveguides," in Proceedings of the Integrated Photonics Research and Applications Topical Meetings (IPRA '05), 2005, paper JWA3.

[21] M. Krause, H. Renner, and E. Brinkmeyer, "Efficiency increase of silicon-on-insulator Raman lasers by reduction of freecarrier absorption in tapered waveguides," in Proceedings of the Conferenceon Lasers and Electro-Optics (CLEO '05), 2005, paper CThB1.

[22] M. Krause, H. Renner, and E. Brinkmeyer, "Analysis of Raman lasing characteristics in silicon-on-insulator waveguides," Optics Express, vol. 12, no. 23, pp. 5703-5710, 2004.

[23] C. Monat, B. Corcoran, D. Pudo et al., "Slow light enhanced nonlinear optics in silicon photonic crystal waveguides," IEEE Journal on Selected Topics in Quantum Electronics, vol. 16, no. 1, pp. 344-356, 2010.

[24] B. Corcoran, C. Monat, D. Pudo et al., "Nonlinear loss dynamics in a silicon slow-light photonic crystal waveguide," Optics Letters, vol. 35, no. 7, pp. 1073-1075, 2010.

[25] C. Monat, B. Corcoran, M. Ebnali-Heidari et al., "Slow light enhancement of nonlinear effects in silicon engineered photonic crystal waveguides," Optics Express, vol. 17, no. 4, pp. 2944-2953, 2009.

[26] X. Checoury, M. El Kurdi, Z. Han, and P. Boucaud, "Enhanced spontaneous Raman scattering in silicon photonic crystal waveguides on insulator," Optics Express, vol. 17, no. 5, pp. 3500-3507, 2009.

[27] J. F. McMillan, M. Yu, D. L. Kwong, and C. W. Wong, "Observation of spontaneous Raman scattering in silicon slow-light photonic crystal waveguides," Applied Physics Letters, vol. 93, no. 25, Article ID 251105, 2008.

[28] Y. Okawachi, M. A. Foster, J. E. Sharping, A. L. Gaeta, Q. Xu, and M. Lipson, "All-optical slow-light on a photonic chip," Optics Express, vol. 14, no. 6, pp. 2317-2322, 2006.

[29] J. F. McMillan, X. Yang, N. G. Panoiu, R. M. Osgood, and C. W. Wong, "Enhanced stimulated Raman scattering in slow-light photonic crystal waveguides," Optics Letters, vol. 31, no. 9, pp. 1235-1237, 2006.

[30] M. Krause, A. Petrov, and E. Brinkmeyer, "Total gain of silicon Raman amplifiers: scaling with group velocity in slow-light waveguides," in Proceedings of the 6th International Conference on Group IV Photonics (GFP '09), 2009, paper ThP5. 
[31] I. D. Rukhlenko, M. Premaratne, and G. P. Agrawal, "Nonlinear silicon photonics: analytical tools," IEEE Journal on Selected Topics in Quantum Electronics, vol. 16, no. 1, pp. 200-215, 2010.

[32] X. Chen, N. C. Panoiu, and R. M. Osgood, "Theory of Ramanmediated pulsed amplification in silicon-wire waveguides," IEEE Journal of Quantum Electronics, vol. 42, no. 2, pp. 160170, 2006.

[33] Q. Lin, O. J. Painter, and G. P. Agrawal, "Nonlinear optical phenomena in silicon waveguides: modeling and applications," Optics Express, vol. 15, no. 25, pp. 16604-16644, 2007.

[34] N. C. Panoiu, J. F. McMillan, and C. W. Wong, "Theoretical analysis of pulse dynamics in silicon photonic crystal wire waveguides," IEEE Journal on Selected Topics in Quantum Electronics, vol. 16, no. 1, pp. 257-266, 2010.

[35] I. D. Rukhlenko and M. Premaratne, "Spectral compression and group delay of optical pulses in silicon Raman amplifiers," Optics Letters, vol. 35, no. 18, pp. 3138-3140, 2010.

[36] I. D. Rukhlenko, M. Premaratne, C. Dissanayake, and G. P. Agrawal, "Continuous-wave Raman amplification in silicon waveguides: beyond the undepleted pump approximation," Optics Letters, vol. 34, no. 4, pp. 536-538, 2009.

[37] I. D. Rukhlenko, M. Premaratne, and G. P. Agrawal, "Effect offree carriers on pump-to-signal noise transfer in silicon Raman amplifiers," Optics Letters, vol. 35, pp. 2343-2345, 2010. 

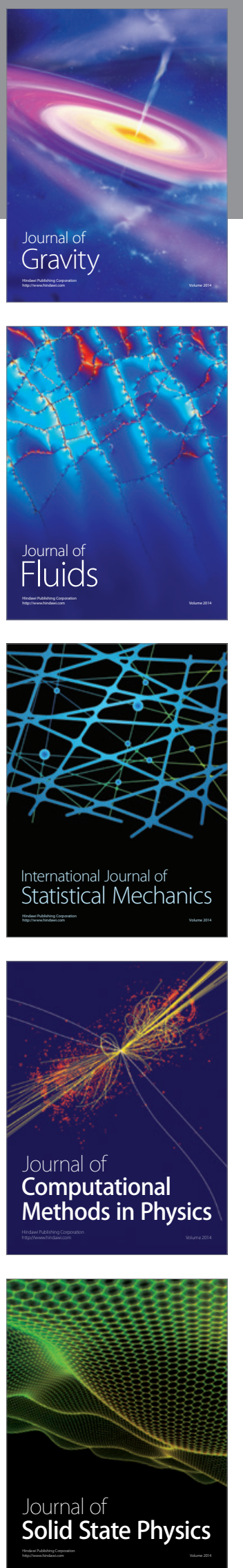

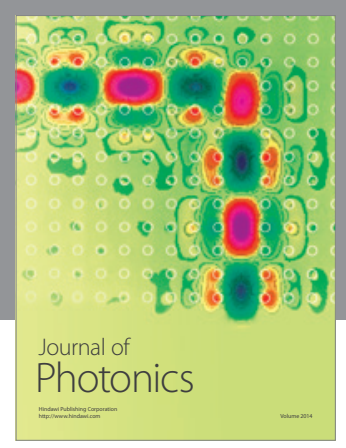

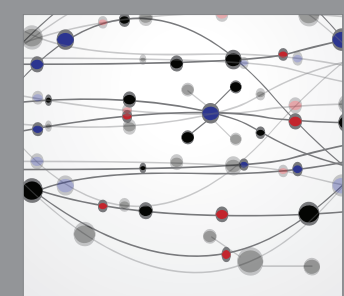

The Scientific World Journal
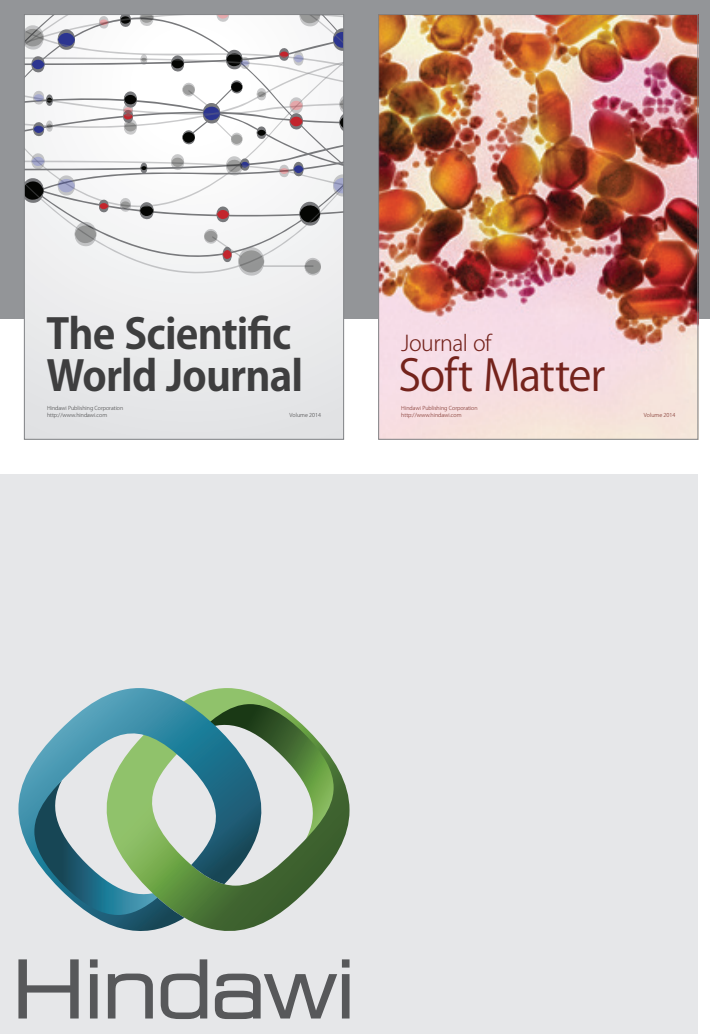

Submit your manuscripts at

http://www.hindawi.com
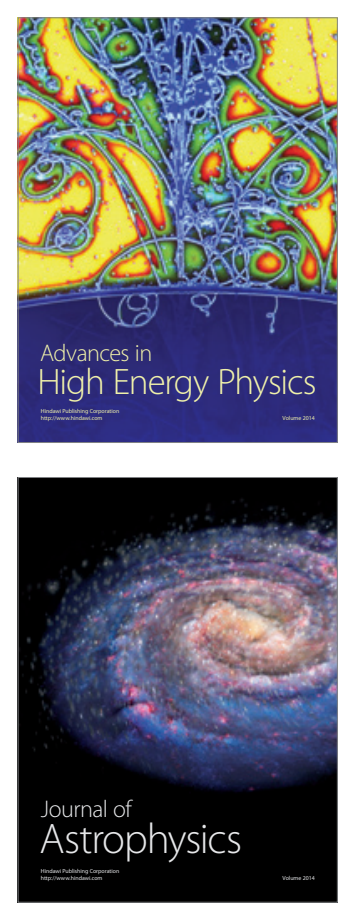
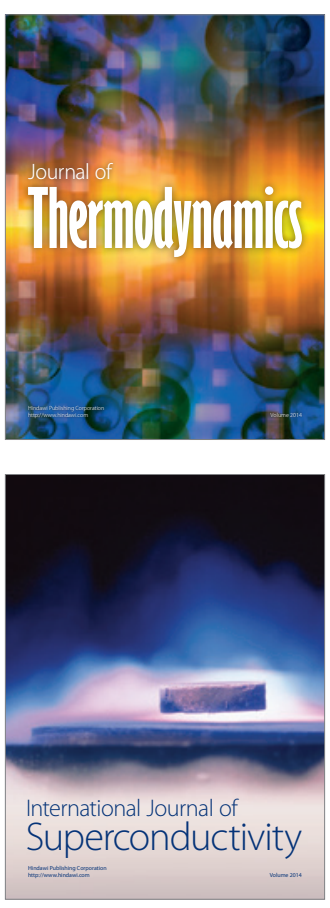
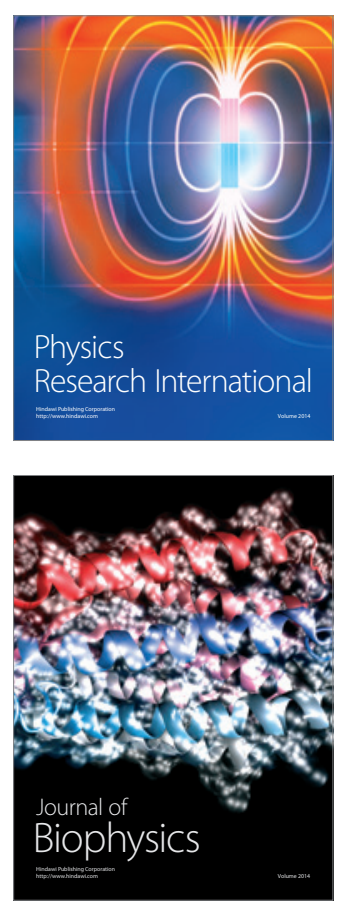
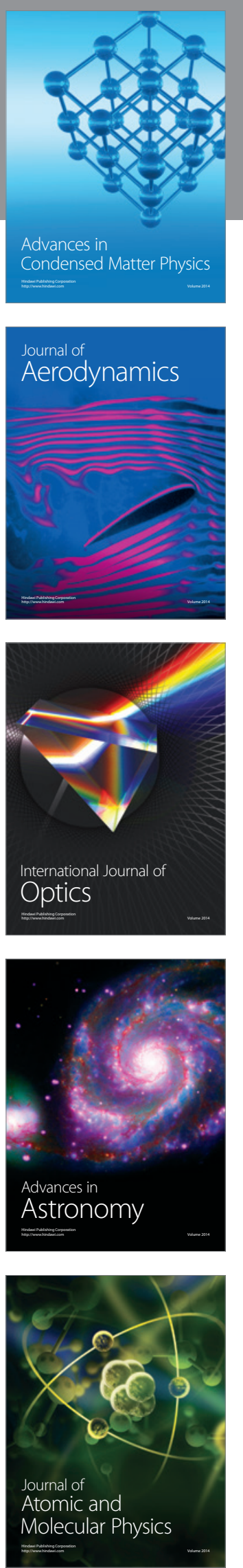